\title{
ESTUDIO DEL RIESGO DE PRESENTAR TRASTORNOS ALIMENTARIOS EN UN GRUPO DE ESTUDIANTES DE LICENCIATURA Y POSGRADO DE INSTITUCIONES MEXICANAS
}

STUDY OF THE RISK OF HAVING EATING DISORDER HABITS

IN UNDERGRADUATE AND GRADUATE STUDENTS IN MEXICAN INSTITUTIONS

\author{
. Paola Pérez Polanco ${ }^{1}$ \\ pperezp@ujsierra.com.mx \\ Luis Manuel Montano Zetina ${ }^{2}$ \\ lmontano@fis.cinvestav.mx
}

1 Escuela de Medicina, Universidad Justo Sierra, México

2 Departamento de Física, Centro de Investigación y de Estudios Avanzados del Instituto Politécnico Nacional, México 
Para citar este artículo:

Pérez P. Paola, Montano Luis. (2020). Estudio del riesgo de presentar trastornos alimentarios en un grupo de estudiantes de licenciatura y posgrado de instituciones mexicanas. Espacio I+D, Innovación más Desarrollo. IX(23), 54-68. doi: http://dx.doi. org/10.31644/IMASD.23.2020.a04

\section{RESUMEN}

El presente trabajo tiene por objetivo detectar la población estudiantil que está en riesgo de presentar trastornos alimentarios (TA). Para ello, se realizó un tamizaje con la aplicación del EAT-26. Los TA y de la ingestión de alimentos con alteraciones persistentes en el comportamiento relacionado con la alimentación, que causa un deterioro significativo de la salud física o del funcionamiento psicosocial de las personas. Los encuestados estuvieron constituidos por 90 estudiantes de Licenciatura en Medicina y, una pequeña población de 20 estudiantes de posgrado. De la población estudiada, se encontró que un $8.2 \%$ de los estudiantes de medicina están en riesgo de presentar TA. De los estudiantes de posgrado no se reportó algún riesgo de TA. Es recomendable que las instituciones realicen programas de intervención que tengan como objetivo la prevención de estos trastornos.

\section{Palabras clave}

Trastornos de la ingesta de alimentos, Bulimia, Anorexia, Dieta restrictiva. 


\section{- Abstract-}

The scope of this work is to study the risk of having Eating disorders (ED) in graduate and postgraduate students in two Mexican institutions. To develop this work, a study of ED prevalence on 90 (20) students from graduate (postgraduate) was performed, using the EAT-16 test. ED represent a major health problem in the population, being responsible for its medial and psychosocial complications. According to the results, after doing data analysis, $8.2 \%$ of the graduate population presented the risk of having or suffering ED. No risk for postgraduate students was detected. Programs to prevent ED in medical institutions are highly recommended.

\section{Keywords}

Eating disorders, Bulimia, Anorexy, Dieting. 
os TA son enfermedades de salud mental complejos, que afectan principalmente a adolescentes y mujeres jóvenes. En el Manual Diagnóstico y Estadístico de los Trastornos Mentales, quinta edición (DSM-5), describe a los TA y de la ingestión de alimentos como una alteración persistente en la alimentación o en el comportamiento relacionado con la alimentación, que produce una alteración en el consumo y que causa un deterioro significativo de la salud física o del funcionamiento psicosocial (APA, 2015).

Los TA son enfermedades que prevalecen más en mujeres jóvenes que en hombres, en una relación 10:1 (López \& Treasure, 2011). Los jóvenes (en especial las mujeres) son los más vulnerables a presentar insatisfacción por su imagen corporal. En un estudio de meta-análisis epidemiológico publicado recientemente sobre los TA en América Latina, reportan una prevalencia de $0.1 \%$ para anorexia nerviosa, $1.16 \%$ para bulimia nerviosa y $3.53 \%$ para el trastorno por atracón (Kolar, Mejía, Mebarak \& Hoek, 2016).

La etiología de este tipo de trastornos es multifactorial. Entre estos factores se pueden mencionar: la preocupación por la figura, la percepción del propio cuerpo, la baja autoestima, el ideal de delgadez imperante, el seguimiento de dietas de adelgazamiento, entre otros. Asimismo, se han reportado factores genéticos (Culbert, Racine \& Klump, 2016; Grzelak, Dutkiewics, Paszynska, Dmitrzak- Weglarz, Slopien \& Tyszkiewicz-Nwafor, 2017) y psicológicos (Geller, Iyar, Kelly \& Srikameswaran, 2019). En estudiantes universitarios, Dávila y colaboradores, han reportado que los TA se ven afectados por problemas psicológicos como baja autoestima, ansiedad, depresión, problemas interfamiliares, carga de trabajo escolar; entre los más representativos (2014). Ruggiero y colaboradores refieren que, en el caso de estudiantes de ciencias de la salud, hay situaciones estresantes que desencadenan los TA como el perfeccionismo patológico (preocupación por errores), insatisfacción corporal, motivación por la delgadez y aumento en las presiones académicas por alcanzar una buena calificación, o bien por aprobar las asignaturas (2008).

El exceso de publicidad en los medios ha repercutido en la imagen que se debe seguir, en cuanto a vestimenta y la forma de actuar para estar a la moda. Kelly Rae Chi, en su artículo publicado en la revista Nature, menciona que "los medios de comunicación masivos ejercen una poderosa influencia en la percepción de la imagen de nuestro cuerpo". Chi concluye; "hay una gran industria allá afuera que no quiere que nos sintamos satisfechos de nosotros" (2015). 
Se tienen varios reportes en cuanto a la prevalencia de los TA en México. En un trabajo realizado por Unikel-Santoncini et al. evaluaron a 4358 mujeres de diferentes estados de la República Mexicana (Aguascalientes, Colima, Chiapas, Chihuahua, Distrito Federal, Durango, Estado de México, Guanajuato, Guerrero, Hidalgo, Jalisco, Michoacán, Nayarit, Nuevo León, Tamaulipas, Oaxaca y Veracruz), encontrando que existe una prevalencia total de riesgo moderado en un $14.2 \%$, y de riesgo alto de $60.8 \%$, de presentar TA (2010). En un estudio realizado en Tijuana, de una muestra de 2322 personas, se reportó que la prevalencia por preocupación por el peso fue del 69.2\%; por la dieta, $24.8 \%$, y el $2 \%$ tenían una prevalencia de TA (Bojorquez, Bustos, Valdez \& Unikel, 2018). En estudiantes de medicina (Morán, Cruz e Iñárritu) reportaron que la población estudiantil de la Licenciatura de Médico Cirujano de la Universidad Nacional Autónoma de México (unAm) presentan una prevalencia de conductas de riesgo de 5.8\%, entre las conductas reportaron un $9.7 \%$ de estudiantes que presentaron eventos de atracones, $5.6 \%$ vómito y $5.6 \%$ uso de laxantes (2009).

La diferente sintomatología y conducta de los pacientes que están pasando por TA, hacen difícil tener un diagnóstico oportuno para poder realizar una intervención inmediata. Es por ello que, en las últimas décadas, se han creado instrumentos que permiten realizar esta tarea. Los principales instrumentos que ayudan a evaluar los TA son los cuestionarios autoaplicables y las entrevistas estructuradas. Entre los cuestionarios autoaplicables están el EAT 40 y el 26 (Garner \& Garfinkel, 1979), CHEAT-26 (Maloney et al., 1989), EDE-Q (Fairburn \& Beglin, 1994), EDI (Garnes et al., 1983), BULIT (Thelen et al., 1991), BITE (Henderson \& Freeman, 1987), QEWP-R (Spitzer et al., 1992), SCOFF (Morgan, Reid \& Lacey, 1999). Aunque ayudan a reportar las conductas de riesgo de TA, ninguno de ellos proporciona un diagnóstico específico, por lo que es necesario que, cuando un paciente obtiene una puntuación que indica riesgo, sea sometido a una entrevista estructurada guiada por un profesionista experto en el manejo del tratamiento.

Con base en lo anterior, el propósito de este trabajo es aplicar el EAT-26, para identificar el riesgo de presentar TA en estudiantes de licenciatura y posgrado. En particular, se seleccionó a un grupo de estudiantes de medicina (90) y a uno de posgrado (20). Ambos grupos de estudio pertenecen a la Ciudad de México. La importancia del presente trabajo radica en reconocer a la población en riego para canalizarla al área correspondiente de la universidad, para así establecer las estrategias de intervención. 


\section{MÉTODO}

\section{Participantes}

Se realizó un muestreo de tipo no probabilístico seleccionado por oportunidad. La muestra estuvo conformada por 90 estudiantes de primero y tercer semestre de la Escuela de Medicina de la Universidad Justo Sierra, junto con 20 estudiantes de posgrado del Departamento de Física del Centro de Investigación y de estudios Avanzados del Instituto Politécnico Nacional (Cinvestav-IPN).

\section{Instrumento}

El instrumento utilizado en este trabajo fue el EAT-26. A nivel internacional el EAT-26 ha sido validado para estimar a la población en riesgo de presentar TA, como anorexia nerviosa, bulimia nerviosa y atracones a diferentes edades, siendo utilizado principalmente en población adolescente (Lazo, Quenaya \& Mayta-Tristán; Wong, Lin \& Chang, 2014). También ha sido aplicado, con buenos resultados, en una población mayor a los 20 años tal como lo muestra el estudio realizado por Harris et al., en el 2015. En el caso de México, el EAT-26 fue traducido, validado y aplicado a una población joven; detectando un porcentaje de población vulnerable a desarrollar TA (Camarillo et al., 2013; Gayou- Esteva \& Ribeiro-Toral, 2014; Morán et al., 2009; Ochoa, 2011). En un trabajo realizado por Álvarez- Rayón et al., encontraron un nivel adecuado de consistencia interna del EAT-26 $(\boldsymbol{\alpha}$ de Cronbach $=0.93)(2004)$. Por esto, este instrumento fue elegido y utilizado para esta investigación.

El EAT-26 es un cuestionario autoaplicable con 26 preguntas y cada una de ellas se responde con una escala tipo Linkert, que va desde siempre hasta nunca. Todas las preguntas, excepto la 25, tienen el siguiente puntaje: o puntos en las respuestas nunca, casi nunca y algunas veces; 1 punto en bastantes veces, 2 puntos en casi siempre y 3 puntos a la respuesta siempre. En la pregunta 25; la puntuación en las respuestas siempre, casi siempre y bastantes veces, tienen un valor de o; algunas veces 1 punto, casi nunca 2 puntos y nunca 3 puntos. El total de puntos del cuestionario puede ir de 0 a 66 puntos. Si el puntaje obtenido es igual o mayor a 20, significa que el encuestado está predispuesto a padecer un TA (Jones, Bennett, Olmsted, Lawson \& Rodin, 2001). Los resultados de las encuestas fueron analizados a través de una base de datos creada en el programa estadístico spss (Statistical Package for the Social Sciences). 


\section{Consideraciones éticas}

Los estudiantes que participaron en este trabajo firmaron un formato de consentimiento informado. En el que se asegura que su participación cumplirá con los lineamientos de la Declaración de Helsinki, y del artículo 100 de la Ley General de Salud (Gobierno de México, 2012) en materia de investigación. Respetando así los principios de protección de privacidad, voluntariedad y dignidad.

\section{RESULTADOS}

Como primer paso, se realizó un estudio sociodemográfico de los estudiantes encuestados. De la muestra total de 110 estudiantes de ambas instituciones, 33.6\% fueron masculinos y 66.4\% femeninos (Figura 1A). El promedio de edad para el caso de los estudiantes de licenciatura fue de 20 años (80\%), para posgrado de 28 (20\%). El 81.8\% se encuentra cursando la licenciatura en Médico Cirujano, el 6.4\% está cursando la maestría en física, y, el 11.8\% el doctorado en Física (Figura 1B).
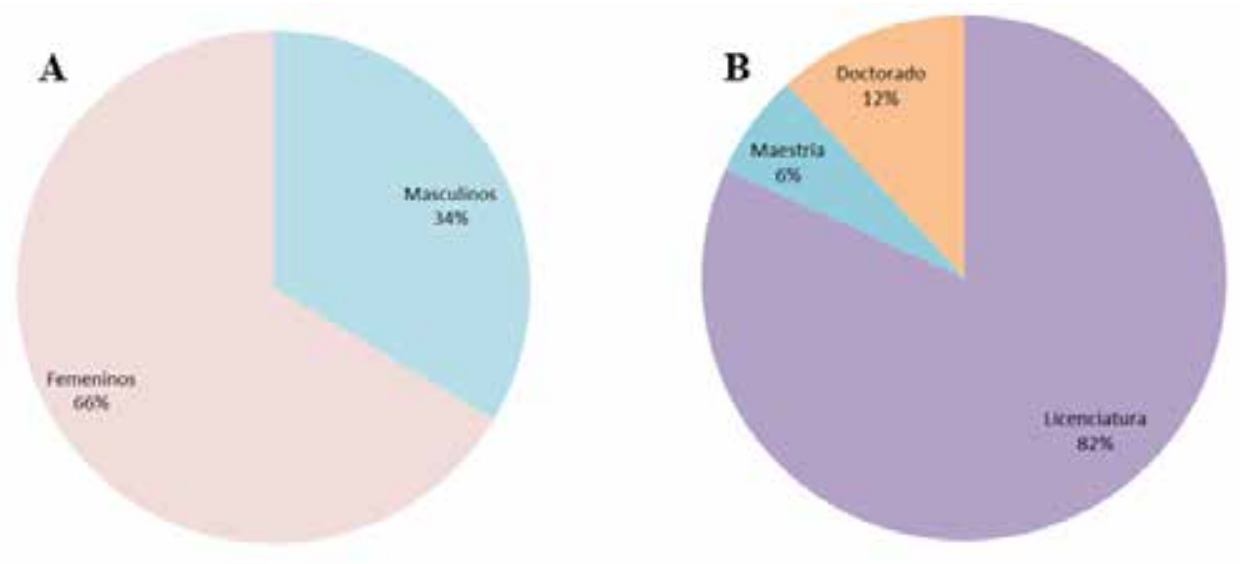

Figura 1. Datos sociodemográficos, A. Género B, grado académico

El segundo paso consistió en obtener el promedio de los puntajes obtenidos de los cuestionarios, que resultó ser de 11.304, con una desviación estándar de 7.187. Se puede observar que los puntajes se encuentran muy dispersos, lo que se asocia a la heterogeneidad del grupo de estudio.

Las preguntas del EAT-26 fueron estructuradas en tres factores: 1, hacer dieta; 2, bulimia y preocupación por la comida; 3, control oral. De acuerdo a las respuestas obtenidas, el $\mathbf{9 1 . 8 \%}$ obtuvo un porcentaje menor a 20, es decir, no presentan riesgos que se asocien a actitudes y conductas alimentarias de riesgo. El 8.2\% de los estudiantes obtuvieron un puntaje mayor a 20 , 
es decir, sus respuestas se asocian a actitudes y conductas alimentarias de riesgo. En cuanto a los puntajes obtenidos en los tres factores mencionados anteriormente se encontró que: el factor 1, lo obtuvo el $20.8 \%$ de los encuestados (13.8\% Mujeres y $7 \%$ Hombres); el factor 2, fue elegido por el 7.2\% (4.8\% Mujeres y 2.4\% Hombres) y finalmente el factor 3, se encontró al $12.3 \%$ de las respuestas de los participantes (8.2\% Mujeres y $4.1 \%$ Hombres); ver figura 2.

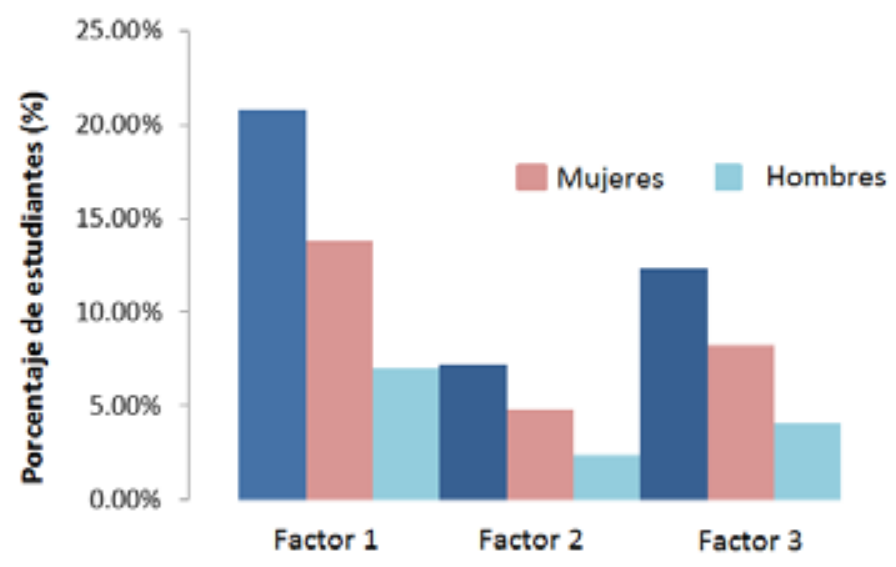

Figura 2. Respuesta de los encuestados en los diferentes factores

En el caso del $8.2 \%$ de los estudiantes que presentaron riesgo de tener TA, pertenecen a la Licenciatura de Medicina, el 6.4\% son mujeres y el 1.8\% son hombres (Figura 3). El 66.6\% obtuvo mayor puntaje en el factor hacer dieta; $\mathbf{1 1 . 2} \%$ en el factor 2 , bulimia; y $22.2 \%$ en el factor 3 que corresponde a control oral.
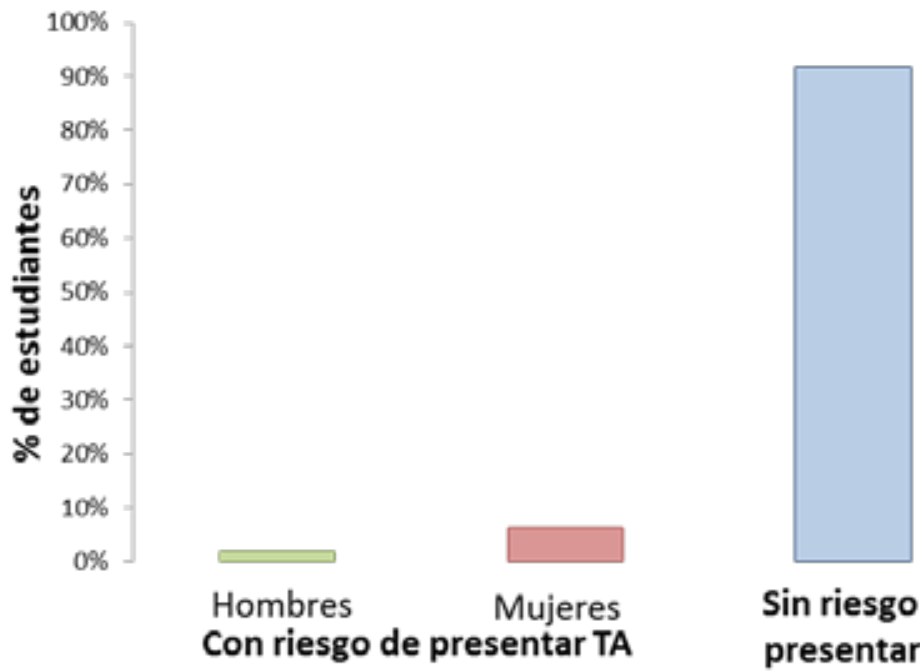

\section{Con riesgo de presentar TA}

\section{Sin riesgo de presentar TA}

Figura 3. Porcentaje de estudiantes con riesgo de presentar TA (Trastornos alimentarios) 
De las estudiantes de posgrado se encontró que, el 25.76\% de sus respuestas corresponde al factor 1 , hacer dieta; el $5.8 \%$ de sus respuestas correspondían al factor 2, bulimia y preocupación por la comida; finalmente el 13.0\% de sus respuestas se encuentra en el factor 3, control oral. Aunque estos resultados tienen el mismo comportamiento que los obtenidos por las estudiantes de pregrado, no se presentó ningún caso de TA.

\section{DISCUSIÓN}

El resultado final de este trabajo fue que el $8.2 \%$ de la población estudiada tiene riesgos de TA. Este resultado es similar al meta-análisis reportado por Jahrami, Sater, Abdulla, Faris \& AlAnsari, quienes encontraron el 10.4\% de riesgo de TA, con una variación de 2.2 al 29.1\% (2019) en las comunidades estudiantiles de medicina. En otro trabajo, realizado por Jahrami, Saif, Faris \& Levine, uno de cada diez estudiantes de medicina corre el riego de presentar TA (2019). Este porcentaje es de severidad, ya que según lo reportado por Keski-Rahkonen \& Mustelin, el 80\% de las personas con este trastorno estarían en riesgo de cursar padecimientos psiquiátricos como trastorno de ansiedad $(<50 \%)$, trastorno afectivo ( $>40 \%)$ y uso de sustancias (>10\%) (2016).

Se ha visto que la población estudiantil de medicina tiene mayor tendencia a desarrollar TA. Debido a que, constantemente, están expuestos al estrés académico, la carga horaria, la necesidad de aprendizaje continuo, la exposición a enfermedades, la muerte de los pacientes durante su educación médica (Rotenstein et al., 2016; Puthran, Zhang, Tam Ho, 2016; Pacheco et al., 2017). El estrés académico que presentan los estudiantes de medicina es reportado como un malestar producido por factores físicos o emocionales, que ejercen una presión significativa, afectando su rendimiento académico y su habilidad meta-cognitiva para resolver problemas. Las exigencias académicas que generan estrés son: las tareas y la falta de tiempo para su realización, la sobrecarga académica, la exposición de trabajos en clase y la realización de exámenes (Zárate et al., 2017; Román y Hernández, 2011).

Por otro lado, autores como Dahlin y colaboradores (2005), así como Uzun y colaboradores (2006), consideran que factores como las bajas calificaciones adquiridas en sus exámenes, las restricciones en las actividades sociales, el sedentarismo, entre otros, son factores desencadenantes para el desarrollo de TA. Todos estos factores contribuyen a que esta población adquiera malos hábitos alimenticios como omitir algunas comidas o, en época de exámenes, realizar una alta ingesta calórica de alimentos poco nutritivos. Sin embargo, uno de los factores que seguirá influyendo en estos trastornos es, sin duda, la edad; debido a que es en la adolescencia media y tardía donde prevalece mayormente los TA. Sabemos que en la adolescencia media existen cambios físicos, mentales, emocionales y sexuales; y en la 
adolescencia tardía es el periodo de crecimiento físico, mental y emocional. En estas etapas se conoce que el 13\% de los jóvenes experimentan al menos un TA, como puede ser principalmente anorexia y bulimia. Este mismo fenómeno se reporta en la edad de 20 años, 15 - 47\%, (Stice, Marti \& Rohde, 2013; Culbert, Racine \& Klump, 2016). La edad en la población estudiada fue de 20 años, es decir, están cursando una adolescencia tardía y son susceptibles a sentir presión por la delgadez, ya sea por la influencia de los medios de comunicación o por la idealización que tienen por la complexión esbelta. Por otro lado, en el grupo de 20 estudiantes de posgrado, tanto de maestría como de doctorado para este estudio, tuvieron una edad promedio de 28 años, ya son considerados como adultos (adulto joven), por lo que el factor edad influye en que no tengan riesgo de TA. Aunque esta muestra de 20 estudiantes no es significativa, nos ayudó a tener un panorama más claro sobre el comportamiento que se tiene en esa edad de presentar TA.

Es aconsejable que las instituciones educativas que detecten en su población estudiantil TA, realicen programas de intervención, que tengan como objetivo la prevención de estos trastornos. Por ejemplo: talleres de habilidades sociales, talleres sobre cómo elaborar menús equilibrados y económicos, importancia del desayuno en la dieta, talleres cognitivo conductuales para manejo de estrés, entre otros. Sobre los talleres cognitivo conductuales, algunos estudios, han reportado la disminución de estrés e incremento en el rendimiento académico en estudiantes que asistieron voluntariamente (Bisson, Ehlers, Matthews, Pilling, Richards \& Turner, 2007; Muñoz \& Pérez, 1997; Richardson \& Rothstein, 2008). En un futuro próximo, se pretende hacer una correlación de factores estresantes en los estudiantes de medicina como causantes de los TA.

El presente estudio tuvo la limitación de contar con un número relativamente pequeño de participantes. En el primer caso, siendo una escuela de medicina del sector privado, la población no fue tan grande como normalmente existen en otras instituciones públicas. En el segundo caso, la población fue aún menor ya que se sabe que existen pocos estudiantes, a diferencia de la población nacional que realizan un posgrado en diferentes áreas científicas. En un futuro se espera extender y aplicar este estudio en una población mayor, abarcando otros factores como los mencionados anteriormente.

\section{CONCLUSIÓN}

Este estudio encontró el riesgo de presentar TA en la población estudiantil de medicina y no se encontró el riesgo en la población de posgrado. De acuerdo a la literatura consultada, misma que sostiene los resultados obtenidos, consideramos que es importante la detección a tiempo de los 
TA. Con la finalidad de actuar de forma inmediata para evitar la cronicidad y lamentables consecuencias, y para el buen desarrollo biopsicosocial del individuo; en particular, de la población con riesgo de TA de este estudio. Se dio el acompañamiento adecuado enviando a los estudiantes afectados al área correspondiente dentro la institución para realizarles una entrevista para establecer o no la presencia de algún padecimiento.

\section{Agradecimientos}

A la Estudiante Nancy Díaz Chávez de la Licenciatura de Médico Cirujano de la Universidad Justo Sierra por apoyar en la aplicación de algunas encuestas. 


\section{BIBLIOGRAFÍA}

Alvarez- Rayón, G., Mancilla-Díaz, J. M., Vázquez-Aré-valo, R., UnikelSantoncini, C., Caballero-Romo, A. \& Mercado-Corona, D. (2004). Validity of the Eating Attitudes Test: A study of Mexican patients with ea-ting disorders. Eating and Weight Disorders, 9, 243-248.

Association, A. (2015). The American Psychiatric Association Practice Guidelines for the Psychiatric Evaluation of Adults. Washington, D.C.: American Psychiatric Publishing.

Bisson, J., Ehlers, A., Matthews, R., Pilling, S., Richards, D., \& Turner, S. (2007). Psychological treatments for chronic post-traumatic stress disorder. British Journal Of Psychiatry, 190(2), 97-104. doi: 10.1192/bjp. bp.106.021402

Bojorquez, I., Bustos, J., Valdez, V., \& Unikel, C. (2018). Life course, sociocultural factors and disordered eating in adult Mexican women. Appetite, 121, 207-214. doi: 10.1016/j.appet.2017.11.085

Camarillo, N., Cabada, E., Gómez, A. \& Munguía, E. (2013). Prevalencia de trastornos de la alimentación en adolescentes. Revista de Especialidades Médico-Quirúrgicas, 51-55.

Chi, K. (2015). Masculinity: Men's makeover. Nature, 526(7572), S12-S13. doi: $10.1038 / 526 s 12 \mathrm{a}$

Culbert, K., Racine, S., \& Klump, K. (2016). Hormonal Factors and Disturbances in Eating Disorders. Current Psychiatry Reports, 18(7). doi: 10.1007/s11920-016-0701-6

Dahlin, M., Joneborg, N., \& Runeson, B. (2005). Stress and depression among medical students: a cross-sectional study. Medical Education, 39(6), 594-604. doi: 10.1111/j.1365-2929.2005.02176.x

Davila, P., Kolodziejczyk, J., Norman, G., Calfas, K., Huang, J., Rock, C., et al. (2014). Relationships between depression, gender, and unhealthy weight loss practices among overweight or obese college students. Eat Behav, 15(2):271-4.

Fairburn, C. \& Beglin, S. (1994). Assessment of eating disorders:interview or self report questionnaire? Inter J Eat Disord. 16, 363-370.

Garner, D. \& Garfinkel, P. (1979). The eating attitudes test: An index of the symptoms of anorexia nervosa. Psychological Medicine, 9, 273-279.

Garnes, D. \& Olmsted, M. (1983). Development and validation of a multidimensional Eating Disorder Inventory for Anorexia Nervosa and Bulimia. Int $J$ Eat Disord. 2, 15-34.

Gayou-Esteva, U., \& Ribeiro-Toral, R. (2014). Eating disorders identification of risk cases among students from Querétaro. Revista Mexicana De Trastornos Alimentarios, 5(2), 115-123. doi: 10.1016/s2007-1523(14)72007-5 
Geller, J., Iyar, M., Kelly, A., \& Srikameswaran, S. (2019). Barriers to selfcompassion in the eating disorders: The factor structure of the fear of self-compassion scale. Eating Behaviors, 35, 101334. doi: 10.1016/j. eatbeh.2019.101334

Grzelak, T., Dutkiewicz, A., Paszynska, E., Dmitrzak-Weglarz, M., Slopien, A., \& Tyszkiewicz-Nwafor, M. (2017). Neurobiochemical and psychological factors influencing the eating behaviors and attitudes in anorexia nervosa. Journal Of Physiology And Biochemistry, 73(2), 297-305. doi: 10.1007/s13105-016-0540-2

Harris, N., Gee, D., d'Acquisto, D., Ogan, D., \& Pritchett, K. (2015). Eating disorder risk, exercise dependence, and body weight dissatisfaction among female nutrition and exercise science university majors. Journal Of Behavioral Addictions, 4(3), 206-209. doi: 10.1556/2006.4.2015.029

Henderson, M. \& Freeman, A. (1987). Self-rating Scale for Bulimia. The BITE. Br J Psychiatry. 150:18-24.

Jahrami, H., Saif, Z., Faris, M., \& Levine, M. (2019). The relationship between risk of eating disorders, age, gender and body mass index in medical students: a meta-regression. Eating And Weight Disorders Studies On Anorexia, Bulimia And Obesity, 24(2), 169-177. doi: 10.1007/ s40519-018-0618-7

Jahrami, H., Sater, M., Abdulla, A., Faris, M., \& AlAnsari, A. (2019). Eating disorders risk among medical students: a global systematic review and meta-analysis. Eating And Weight Disorders - Studies On Anorexia, Bulimia And Obesity, 24(3), 397-410. doi: 10.1007/s40519-018-0516-Z

Jones, J., Bennett, S., Olmsted, M., Lawson, M. \& Rodin, G. (2001). Disordered eating attitudes and behaviors in teenaged girls: A schoolbased study. Canadian Medical Association Journal, 165, 547-552.

Keski-Rahkonen, A., \& Mustelin, L. (2016). Epidemiology of eating disorders in Europe. Current Opinion In Psychiatry, 29(6), 340-345. doi: 10.1097/ yco.0000000000000278

Kolar, D., Rodriguez, D., Chams, M., \& Hoek, H. (2016). Epidemiology of eating disorders in Latin America. Current Opinion In Psychiatry, 29(6), 363-371. doi: 10.1097/yco.0000000000000279

Lazo, Y., Quenaya, A. \& Mayta-Tristán, P. (2014). Viewing competition reality shows and risk of eating disorders in adolescent schoolchildren. Rev Chil Pediatr. 86(1), 66-67.

López C., Treasure J, (2011). Trastornos de la conducta alimentaria en adolescentes: descripción y manejo. Rev. Med. Clin. Condes, 22(1) 85-97.

Maloney, M., McGuire, J., Daniels, S. \& Specker, B. (1989). Dieting behavior and attitudes in children. Pediatrics. $84,482-489$. 
Morán, I., Licea, V. \& Iñárritu, M. (2009). Prevalencia de factores y conductas de riesgo asociados a trastornos de la alimentación en universitarios. Revista Médica Del Hospital General de México, 72(2), 68-72.

Morgan, J., Reid, F. \& Lacey, H. (1999). The scoff questionnaire: assessment of a new screening tool for eating disorders. BMJ. 319, 1467-1468.

Muñoz, M. \& Pérez, E. (1997). "Eficacia del entrenamiento en inoculación de estrés en el control de la ansiedad. Ansiedad y Estrés, 3, pp. 225-244.

Ochoa, S. (2011). La actitud hacia la comida en estudiantes universitarios: validación y confiabilidad del eating attitudes test (EAT-26). En J. C. Sánchez, \& M. E. Villarreal (Eds.), Tópicos de psicología en la salud en el ámbito universitario (pp. 35-46) Nuevo León, Monterrey: Universidad Autónoma de Nuevo León.

Pacheco, J., Giacomin, H., Tam, W., Ribeiro, T., Arab, C., Bezerra, I., \& Pinasco, G. (2017). Mental health problems among medical students in Brazil: a systematic review and meta-analysis. Revista Brasileira De Psiquiatria, 39(4), 369-378. doi: 10.1590/1516-4446-2017-2223

Puthran R, Zhang мwв, Tam ww, Ho RC (2016) Prevalence of depression amongst medical students: a meta-analysis. Med Educ 50:456-468. https://doi.org/10.1111/medu.12962

Richardson, K., \& Rothstein, H. (2008). Effects of occupational stress management intervention programs: A meta-analysis. Journal Of Occupational Health Psychology, 13(1), 69-93. doi: 10.1037/1076-8998.13.1.69

Román, C., Hernández, Y. (2011). El estrés académico: una revisión crítica del concepto desde las ciencias de la educación. Revista Electrónica de Psicología Iztacala, 14: 1-14.

Rotenstein, L., Ramos, M., Torre, M., Segal, J., Peluso, M., \& Guille, C. et al. (2016). Prevalence of Depression, Depressive Symptoms, and Suicidal Ideation Among Medical Students. JAMA, 316(21), 2214. doi: 10.1001/ jama.2016.17324

Ruggiero, G., Bertelli, S., Boccalari, L., Centorame, F., Ditucci, A., La Mela, C., et al. (2008) The influence of stress on the relationship between cognitive variables and measures of eating disorders (in healthy female university students): A quasi experimental study. Eat Weight Disord, 13 (3): $142-8$.

Spitzer, R., Devlin, M., Walsh, B., Hasin, D., Wing, R., Marcus, M., Stunkard, A., Wadden, T., Yanovski, S., Agras, S. \& Nonas, C. (1992). Binge eating disorder: A multi-site field trial of the diagnostic criteria. Int $J$ Eat Disord. 11:191-203.

Stice, E., Marti, C., \& Rohde, P. (2013). Prevalence, incidence, impairment, and course of the proposed DSM-5 eating disorder diagnoses in an 8 -year prospective community study of young women. Journal of Abnormal Psychology, 122(2), 445-457. doi: 10.1037/a0030679 
Thelen, M., Farmer, J., Wonderlich, D. \& Smith, M. (1991). A revision of the bulimia test: the BULIT-R. J Consult Clin Psychol. 3, 119-124.

Unikel- Santoncini, C., Nuño-Gutiérrez, B., Celis-de la Rosa, A., SaucedoMolina, T., Trujillo, E. \& García-Castro, F. (2010). Conductas alimentarias de riesgo: prevalencia en estudiantes mexicanas de 15 a 19 años. Revista de Investigación Clínica, 62, 424-432.

Uzun, Ö., Güleç, N., Özşahin, A., Doruk, A., Özdemir, B., \& Çalişkan, U. (2006). Screening disordered eating attitudes and eating disorders in a sample of Turkish female college students. Comprehensive Psychiatry, 47(2), 123-126. doi: 10.1016/j.comppsych.2005.05.004

Wong, Y., Lin, J.S., Chang, Y.J. (2014). Body satisfaction, emotional intelligence, and the development of disturbed eating: a survey of Taiwanese students. Asia Pac J Clin Nutr, 23(4):651-9.

Zárate, N., Soto, M., Castro, M., Quintero, J. (2017) Estrés académico en estudiantes universitarios: medidas preventivas. Revista de la Alta Tecnología y la Sociedad, 9: 92-8. 